\title{
Shape and Size of Giant Unilamellar Phospholipid Vesicles Containing Cardiolipin
}

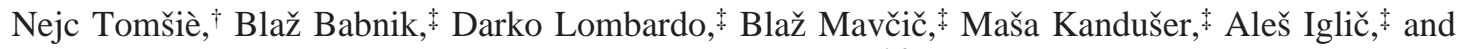 \\ Veronika Kralj-Iglič $*,+, \S$
}

Faculty of Medicine, University of Ljubljana, Vrazov trg 2, Ljubljana, Slovenia, Faculty of Electrical Engineering, University of Ljubljana, Tržaška 25, Ljubljana, Slovenia, and Nomadic College, C/Berlanger, Rue Esseghem 2, 1090 Brussels, Belgium

Received May 8, 2005

\begin{abstract}
The effect of cardiolipin content on the shape and size of giant palmitoyloleylphosphatidylcholine/cardiolipin vesicles was studied. Unilamellar vesicles were prepared in sugar solution by the method of electroformation, from mixtures containing up to 50\% weight ratio of cardiolipin. At room temperature the vesicles containing cardiolipin exhibited abrupt changes in the curvature of the vesicle contour indicating regions of phase separation. The deviations from the spherical shape were larger if vesicles were made from mixtures with a higher content of cardiolipin. Numerous vesicles with soft fluctuating walls were observed. The estimated size of the vesicles containing cardiolipin was found to be smaller than the size of pure palmitoyloleylphosphatidylcholine vesicles.
\end{abstract}

\section{INTRODUCTION}

Cardiolipin is a four-tailed phospholipid, present mostly in the inner leaflet of the mitochondrial membrane. Membranes containing cardiolipin interact with anticardiolipin antibodies. ${ }^{1}$ Anticardiolipin antibodies belong to a heterogeneous group of antiphospholipid antibodies; in clinical practice, the presence of anticardiolipin antibodies in the blood serum is one out of two formal serological criteria sufficient to confirm the antiphospholipid syndrome ${ }^{2}-a$ disorder that is connected to vascular thrombosis.

Giant unilamellar phospholipid vesicles present a convenient model for study of the cell membrane. The properties of the membrane and its constituents are reflected in the vesicle shape that can be observed by an optical microscope. Of special interest is the interaction between cardiolipin and anticardiolipin antibodies which could be studied by observing its effect on the shape and fluctuation pattern of giant unilamellar phospholipid vesicles containing cardiolipin. For that, the properties of cardiolipin containing vesicles should be understood. However, to our knowledge, the effect of cardiolipin on the shape and size of giant phospholipid vesicles has not yet been systematically explored. In this work we observed populations of giant phospholipid vesicles made of different mixtures of palmitoyloleylphosphatidylcholine (POPC) and cardiolipin.

\section{METHODS AND MATERIALS}

Giant phospholipid vesicles were prepared at room temperature $\left(23^{\circ} \mathrm{C}\right)$ by the modified electroformation method. ${ }^{3}$ Synthetic lipids, POPC (1-palmitoyl-2-oleoyl-sn-glycero-3phosphocholine) and cardiolipin (1,1'2,2'-tetraoleoyl cardio-

* Corresponding author phone: +386-1-543-7600; e-mail: vera.kraljiglic@ biofiz.mf.uni-lj.si. Corresponding author address: Institute of Biophysics, Faculty of Medicine, University of Ljubljana, Slovenia.

${ }^{\dagger}$ Faculty of Medicine.

Faculty of Electrical Engineering.

$\S$ Nomadic College. lipin), were purchased from Avanti Polar Lipids, Inc. Adequate volume proportions of POPC and cardiolipin, both dissolved in 2:1 chloroform/methanol in a concentration of $1 \mathrm{mg} / \mathrm{mL}$, were combined in a glass jar and thoroughly mixed. It is assumed that the volume proportions of both phospholipids in the mixture represent their weight ratio in vesicle membranes. Then, $20 \mu \mathrm{L}$ of POPC and cardiolipin mixture was applied to platinum electrodes. The solvent was allowed to evaporate in low vacuum for $30 \mathrm{~min}$. The coated electrodes were placed in the electroformation chamber, and $3 \mathrm{~mL}$ of a $0.2 \mathrm{M}$ sucrose solution was poured into the chamber. An AC electric current with an amplitude of $5 \mathrm{~V}$ and a frequency of $10 \mathrm{~Hz}$ was applied to the electrodes for $2 \mathrm{~h}$, which was followed by $2.5 \mathrm{~V}$ and $5 \mathrm{~Hz}$ for $15 \mathrm{~min}, 2.5$ $\mathrm{V}$ and $2.5 \mathrm{~Hz}$ for $15 \mathrm{~min}$, and finally $1 \mathrm{~V}$ and $1 \mathrm{~Hz}$ for 15 min. The electroformation was observed under an inverted microscope Olympus CK40 with phase contrast optics. The content was rinsed out of the electroformation chamber with $5 \mathrm{~mL}$ of a $0.2 \mathrm{M}$ glucose solution. Immediately after the electroformation, the solution containing vesicles was placed into the observation chamber made from a pair of cover glasses sealed with grease. Vesicles were observed under an inverted microscope Zeiss Axiovert 200 with phase contrast optics. The electroformation and the vesicle shapes were recorded by the Sony XC-77CE video camera. Snapshots of video records were taken in order to measure dimensions of vesicles with the computer program Image Tool. The vesicle size was represented by the diameter of the effective circle. The diameter of the effective circle was approximated by an average of the vertical and the horizontal dimension of the vesicle (Figure 1).

Populations of the vesicles were compared by descriptive statistical methods. Three independent experiments starting from electroformation were performed for each weight ratio of POPC/cardiolipin. The size distributions were considered to be normal. The pooled two-tailed $t$-test was used to compare the average values of the diameters of the effective circles. 


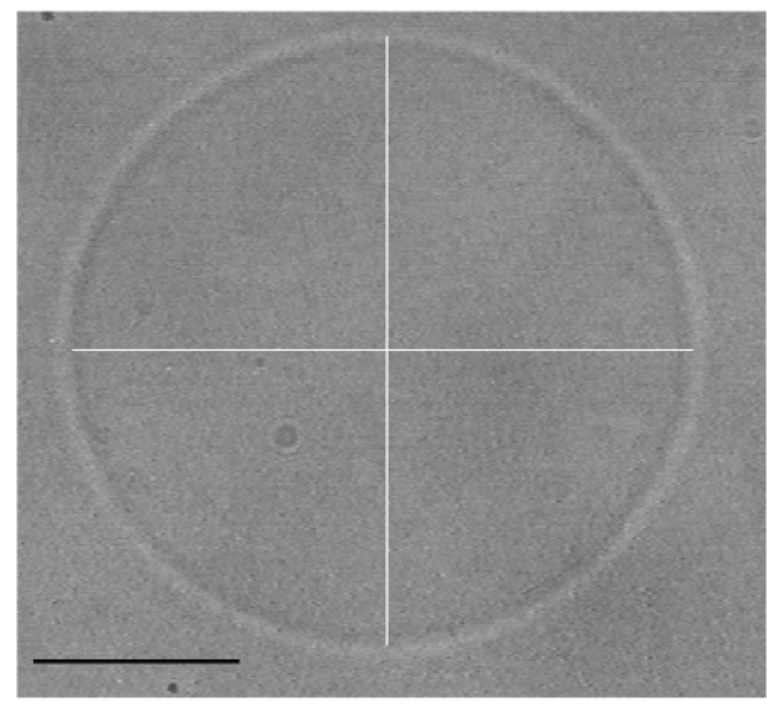

Figure 1. Determination of the vesicle size. Diameter of the effective circle was taken as the average of the vertical and the horizontal dimension of the vesicle. Bar represents $10 \mu \mathrm{m}$.

\section{RESULTS}

We have observed populations of cardiolipin containing POPC vesicles prepared from up to $50 \%$ weight ratio of cardiolipin. Populations prepared from $10 \%$ to $30 \%$ of cardiolipin were abundant in vesicles, while there were significantly less vesicles in populations prepared from $40 \%$ and $50 \%$ of cardiolipin.

The process of electroformation starts with folding and rearrangement of multilayers to yield unilamellar structures. ${ }^{3}$

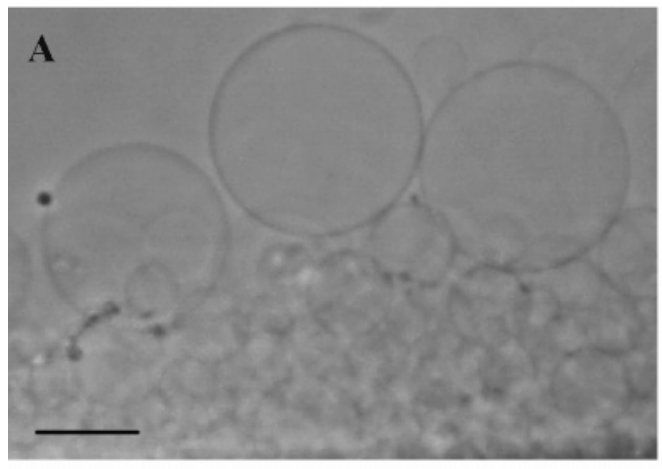

Differences in membrane folding between the sample with pure POPC and the mixture of POPC and cardiolipin could be observed throughout the process. Pure POPC membranes were mostly arranged into spherelike segments (Figure 2A). Cardiolipin containing membranes at the beginning of the process formed elongated structures with bulby ends (Figure 2B). After being rinsed into the plastic beaker and placed into the observation chamber, separated vesicles exhibited the abrupt changes in contour curvature (Figure 3 ) that are visible already later in the electroformation process. The changes in curvature smoothed out if the sample was heated above $40{ }^{\circ} \mathrm{C}$. Upon recooling the abrupt changes in contour curvature reappeared. In the samples prepared from the mixture containing cardiolipin we found $10-50 \%$ vesicles with abrupt changes in contour curvature. The vesicles prepared from the mixture containing a higher content of cardiolipin exhibited larger areas of "nonspherical" shape (Figure 3). We have observed numerous vesicles with soft fluctuating walls (Figure 4).

Populations of vesicles, containing a different content of cardiolipin, have been compared in size. Average sizes of all the vesicles created from pure POPC were found to be the largest (average effective diameter $16.03 \mu \mathrm{m}$, standard deviation (SD) $9.24 \mu \mathrm{m}$ ). The average diameters of the populations containing cardiolipin were smaller than in the vesicles made of pure POPC: for $90 \%$ POPC/10\% cardiolipin the average diameter was $11.51 \mu \mathrm{m}(\mathrm{SD}=6.22 \mu \mathrm{m})$, for $80 \%$ POPC $/ 20 \%$ cardiolipin the average diameter was $12.79 \mu \mathrm{m}(\mathrm{SD}=8.06 \mu \mathrm{m})$, and for $70 \% \mathrm{POPC} / 30 \%$ cardiolipin the average diameter was $11.23 \mu \mathrm{m}(\mathrm{SD}=8.42$

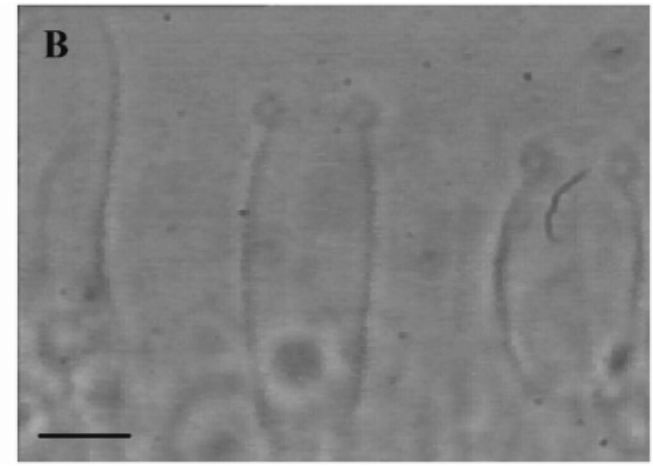

Figure 2. Electroformation of giant phospholipid vesicles: (A) pure POPC membrane and (B) POPC-cardiolipin membrane several minutes after applying the electric field (peculiar owl-like shapes can be distinguished). Bar represents $20 \mu \mathrm{m}$.
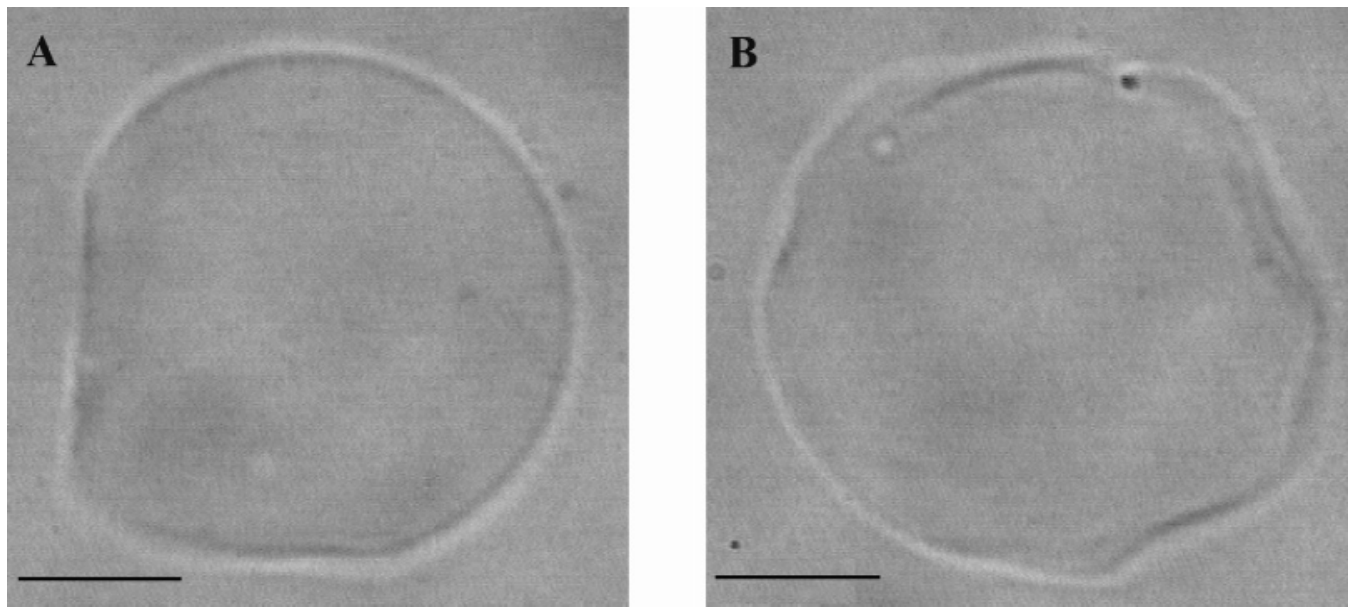

Figure 3. Two giant POPC vesicles prepared from the mixture containing $30 \%$ of cardiolipin that exhibit large areas of "nonspherical" shape. Bar represents $10 \mu \mathrm{m}$. 


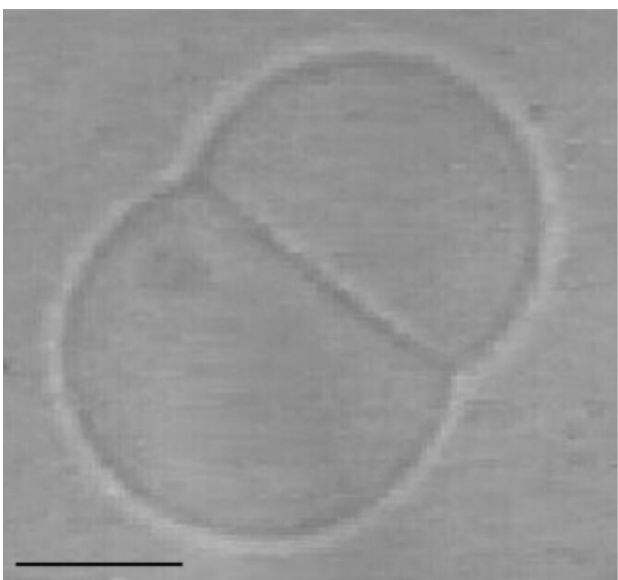

Figure 4. A two-compartment vesicle with a soft bilayer wall between the compartments. Bar represents $10 \mu \mathrm{m}$.

Table 1. Statistical Significance of the Differences in Sizes of Vesicles Prepared from Mixtures Containing Different Weight Ratios of Cardiolipin (CL)

\begin{tabular}{lcccc}
\hline & & $90 \% \mathrm{POPC}$, & $80 \% \mathrm{POPC}$, & $70 \% \mathrm{POPC}$, \\
& $100 \%$ POPC & $10 \% \mathrm{CL}$ & $20 \% \mathrm{CL}$ & $30 \% \mathrm{CL}$ \\
\hline $100 \%$ POPC & $<10^{-5}$ & $<10^{-5}$ & $<10^{-5}$ \\
$90 \%$ POPC & & 0.007 & 0.57 \\
$80 \%$ POPC & & & 0.007 \\
\hline
\end{tabular}

$\mu \mathrm{m})$. The differences between the average sizes of the pure POPC vesicles and the vesicles containing cardiolipin were statistically significant $\left(p<10^{-5}\right.$, Table 1 ). The differences between the average sizes of the vesicles containing a

\section{A Size distribution of vesicles with} $100 \%$ POPC

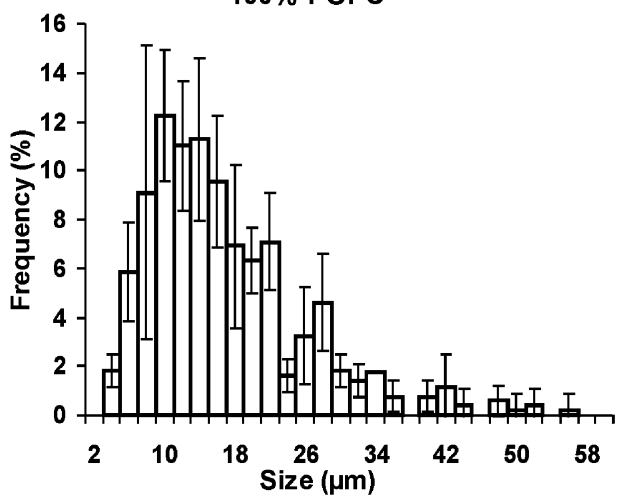

\section{Size distribution of vesicles with $80 \%$} POPC and $20 \%$ cardiolipin

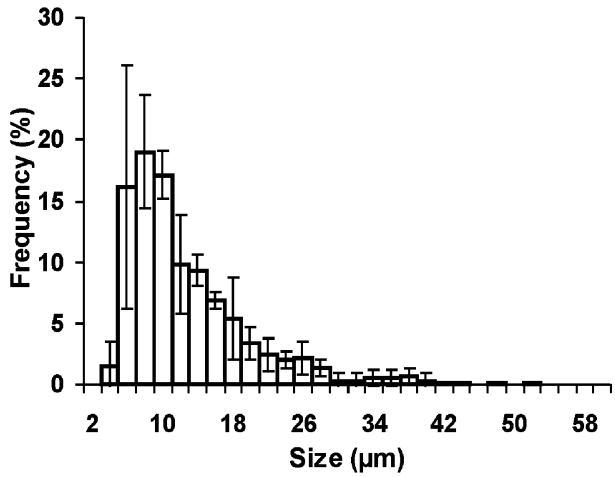

different content of cardiolipin were smaller and statistically less significant than the differences between the average sizes of the pure POPC vesicles and the vesicles containing cardiolipin (Table 1).

\section{DISCUSSION}

Our results indicate that the presence of cardiolipin in the membrane affects both the shape and size of vesicles. In the early stages of electroformation, peculiar elongated shapes resembling an owl are formed. The two-dimensional cut resembling owl eyes could correspond to a bulby toroidlike structure. As cardiolipin has four tails this could correspond to anisotropy of the molecule with respect to the direction perpendicular to the membrane. It was found ${ }^{4,5}$ that such anisotropic molecules favor membrane regions with strongly different principal membrane curvatures. However, later in the process, vesicles attain globular shape with abrupt changes in the contour curvature. Upon heating over $40{ }^{\circ} \mathrm{C}$ the contour of the vesicle becomes smooth. This transition in shape could be explained by crossing the temperature of the main gel-to-liquid crystal-phase transition. Our observations correspond to the previous results ${ }^{6,7}$ where the temperature of Na salt of cardiolipin was determined to be 39.7 ${ }^{\circ} \mathrm{C}$. When the sample was allowed to cool, the abrupt changes in contour curvature reappeared.

It was also found that nanotubular protrusions were attached to cardiolipin containing vesicles, similar as observed in pure phospholipid vesicles. ${ }^{8,9}$ Although the population of the vesicles is heterogeneous regarding their shape and dynamics, characteristical shapes were observed in

\section{B Size distribution of vesicles with $90 \%$ POPC and $10 \%$ cardiolipin}

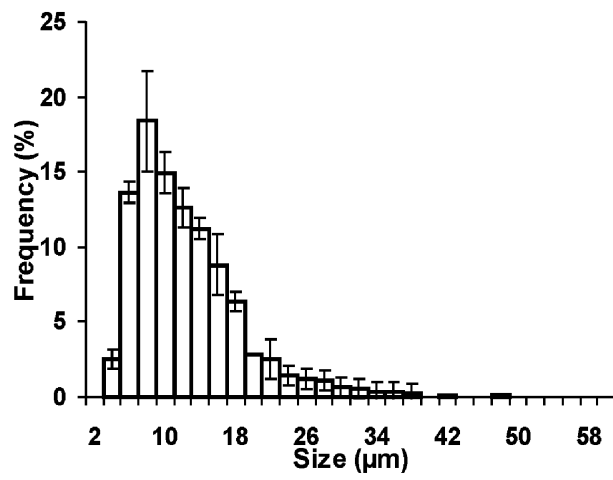

\section{Size distribution of vesicles with $70 \%$} POPC and $30 \%$ cardiolipin

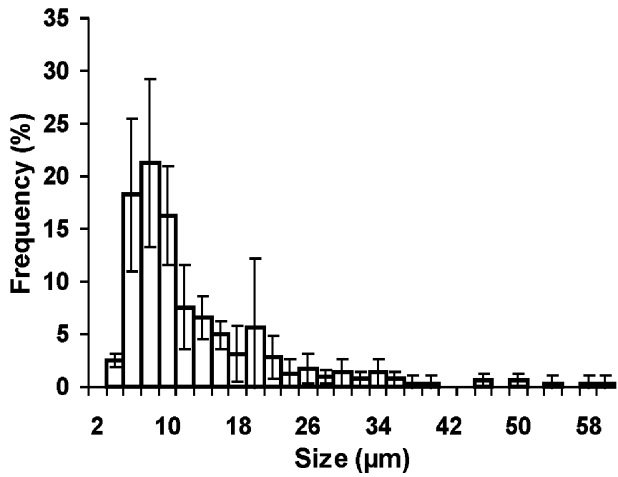

Figure 5. Size (effective vesicle diameter) distribution prepared from mixtures containing different weight ratios of cardiolipin. Errors of each column are estimated respectively as $2 / 3$ of the largest deviation from the average obtained from three independent experiments. 
cardiolipin-POPC vesicles. These shapes appear as manycompartment structures, compartments being separated by soft fluctuating walls (Figure 4). In spontaneous shape transformation the proportion between the area of the outer membrane and the wall membrane changed. If the area of the wall increased with respect to the area of the outer membrane, undulation of the wall appeared. We have observed that the spontaneous transformation leads to the formation of a spherical cavity within one of the compartments divided by the wall. Also shapes composed of many compartments were observed. The appearance of the wall membrane indicates that the wall bifurcates at the ring defining the edge. At the edge, both layers of the wall exhibit a large difference between the principal curvatures that could be favored by an anisotropic structure of cardiolipin molecules.

Vesicles with cardiolipin were found to be smaller than those created from pure POPC. To critically discuss the results on the average size of populations we have to consider that the effective circle representing the size of the vesicle is overestimated if the vesicle shape deviates from the sphere. Vesicles containing cardiolipin are not spherical, the deviation from the sphere being larger for higher content of cardiolipin. Therefore, accuracy of determination of the average size decreases with increasing the cardiolipin content. It should also be considered that there may be "nonspherical" regions that cannot be seen in the contour as the vesicle is a three-dimensional object where such segments may be positioned in the regions that do not correspond to the contour revealed by the micropscope focus. The results shown in Figure 5 and Table 1 should therefore be considered as an upper limit for the average vesicle size. Nevertheless, all populations containing cardiolipin were found to be smaller than pure POPC vesicles. This may indicate a preference of cardiolipin for larger average curvature. Accordingly, large $(<0.1 \mu \mathrm{m})$ multilamellar vesicles of pure cardiolipin were found to be stable. ${ }^{7}$

Analysis of fluctuations of giant phospholipid vesicles could give important information on the effects of the interaction of membrane constituents (cardiolipin) with exogeneously added molecules (antiphospholipid antibodies).
To perform such analysis, it is convenient that the vesicles are unilamellar. Study of different methods for producing phospholipid vesicles showed that electroformation method gives the highest yield of unilamellar vesicles (approximately $95 \%$ ) with a narrow size distribution. ${ }^{10}$ Therefore, electroformation seems to be an appropriate method to produce giant vesicles for investigation of interaction between membrane constituents and exogeneously added molecules by analysis of the vesicle shapes and shape fluctuations.

\section{REFERENCES AND NOTES}

(1) Hatch, G. M. Cardiolipin: biosynthesis, remodeling and trafficking in the heart and mammalian cells (Review). Int. J. Mol. Med. 1998, $1,33-41$.

(2) Wilson, W. A.; Gharavi, A. E.; Koike, T.; Lockshin, M. D.; Branch, D. W.; Piette, J. C.; Brey, R.; Derksen, R.; Harris, E. N.; Hughes, G. R.; Triplett, D. A.; Khamashta, M. A. International consensus statement on preliminary classification criteria for definite antiphospholipid syndrome: report of an international workshop. Arthritis Rheum. 1999, 42, 1309-1311.

(3) Angelova, M. I.; Soléau, S.; Meléard, P.; Faucon, J. F.; Bothorel, P. Preparation of giant vesicles by external AC electric fields. Kinetics and applications. Prog. Colloid Polym. Sci. 1992, 89, 127-131.

(4) Bobrowska-Hägerstand, M.; Kralj-Igliè, V.; Igliè, A.; Bialkowska, K.; Isomaa, B.; Hägerstrand, $\mathrm{H}$. Torocyte membrane endovesicles induced by octaethyleneglycol dodecyl ether in human erythrocytes. Biophys. J. 1999, 77, 3356-3362.

(5) Kralj-Iglič, V.; Iglič, A.; Hägerstrand, H.; Peterlin, P. Stable tubular microexovesicles of the erythrocyte membrane induced by dimeric amphiphiles. Phys. Rev. E 2000, 61, 4230-4234.

(6) Rainier, S.; Jain, M. K.; Ramirez, F.; Ioannou, P. V.; Marecek, J. F.; Wagner, R. Phase transition characteristics of diphosphatidylglycerol (cardiolipin) and stereoisomeric phosphatidyldiacylglycerol bilayers Mono- and divalent metal ion effects. Biochim. Biophys. Acta 1979, 558, 187-198.

(7) Khan, T. K. Binding of hexokinase enzyme to cardiolipin vesicle: influence of hydrophobic matching. Colloids Surf., B 1996, 7, 145151.

(8) Mathivet, L.; Cribier, S.; Devaux, P. F. Shape changes and physical properties of giant phospholipid vesicles prepared in the presence of an AC electric field. Biophys. J. 1996, 70, 1112-1120.

(9) Kralj-Iglič, V.; Gomišček, G.; Majhenc, J.; Arrigler, V.; Svetina, S. Myelin-like protrusions of giant phospholipid vesicles prepared by electroformation. Colloids Surf. A 2001, 181, 315-318.

(10) Bagatolli, L. A.; Parasassi, T.; Gratton, E. Giant phospholipid vesicles: comparison among the whole lipid sample characteristics using different preparation methods: a two photon fluorescence microscopy study. Chem. Phys. Lipids 2000, 105, 135-147.

CI050188B 